\title{
DOs and DON'Ts in Ophthalmology during the COVID-19 Pandemic
}

\author{
Reeda Bou Said \\ Department of Ophthalmology, Lebanese University, Beirut, Lebanon \\ Corresponding Author: Reeda Bou Said, reeda.bousaid@ijcrcentral.com \\ Funding: None \\ Competing Interests: None \\ Ethical approval: Not required
}

\begin{abstract}
Abbreviations: SARS-CoV-2: Severe Acute Respiratory Syndrome Coronavirus 2; COVID-19: coronavirus disease 2019; AAO: American Academy of Ophthalmology; CDC: Centers for Disease Control and Prevention; IOP: Intraocular-pressure; CL: Contact lens; PCR: Polymerase Chain Reaction; BSS: Balanced Salt Solution
\end{abstract}

The recent pandemic caused by SARS-CoV-2 has spanned continents, races, and social constructs. It has caused mortality and morbidity around the world and left those unaffected, or unknowingly so, with constant anxiety from contracting the virus by one means or another. As such, habits have changed, roads have emptied, and hospitals have been overwhelmed. One of the first whistleblowers who tried to alert the medical community to a possible new outbreak was a young ophthalmologist in Wuhan, China, who unfortunately contracted the disease and died in February 2020. The following editorial aims to improve care in ophthalmology during the pandemic.

Due to the close examination and the high viral load in the upper respiratory tract, ophthalmologists and otorhinolaryngologists are at a higher risk of infection with SARS-CoV-2. Conjunctivitis is only reported in $0.8 \%$ of patients with COVID-19, but it may be the presenting symptom in pediatric inflammatory multisystem syndrome [1]. Other nonspecific ocular symptoms associated with COVID-19 include pruritus, lacrimation, secretions, and foreign body sensation [2]. Initial data published by the AAO documented the presence of the virus in the tear film of patients diagnosed with pneumonia and associated conjunctivitis due to SARS-CoV-2. A recent prospective study showed the absence of the virus in 64 tear samples from 17 COVID-19 positive patients who had no ocular disease and one with evidence of injection and chemosis [3]. In our practice, we are frequently attending patients remotely or receiving patients somehow later than usual. Here are some tips for both patients and physicians.

\section{For patients}

- Try calling. Check if your hospital provides telemedicine. Contact your doctor and explain your symptoms, including their time-frame, recent changes, recent trauma, and any relevant systemic manifestations. Slit-lamp examination is crucial for diagnosis in ophthalmology. However, in some cases, such as in mild conjunctivitides and subconjunctival hemorrhages, we can establish a diagnosis based on a description of symptoms and images. As such, observation or preliminary treatment can be attempted. Other symptoms might require a more urgent consultation. 
- Wear a mask. If surgical masks are unavailable or are reserved for medical personnel, you can make a cloth mask as recommended by the CDC (https://www.cdc.gov/coronavirus/2019-ncov/preventgetting-sick/how-to-make-cloth-face-covering.html). Masks may cloud your vision by fogging your eyeglasses if you are wearing a pair. You can avoid this by keeping your glasses fixed to your nose bridge by tape. You can also wash the lenses with soapy water and then let air-dry or use a clean cloth to do so.

- Report any possibility of transmission over the phone, prior to your visit. Inform about any symptom you may be having. Report any recent travel history or contact with suspected or confirmed cases. Ocular symptoms that also need specifying are redness and secretions.

- Comply with drops. If you are on IOP lowering drops, compliance is key. Discuss with your doctor on optimal timing for monitoring IOP in order to limit visits. Regarding other ocular drops, make sure to consult your ophthalmologist.

- Avoid contact lenses. There is no established risk of infection with the use of CL wear. The usual standard of care for $\mathrm{CL}$ wear is always relevant: practice safety and hygiene while handling the lenses, avoid wearing them for prolonged periods, and avoid touching or rubbing your eyes. These practices are of even higher importance today due to the potential risk of transmission of coronavirus. Because contact-lens wearers tend to touch their eyes more frequently, experts have recommended opting for eyeglasses whenever possible, which can also be of partial protection [4]. Any sign of ocular infection should prompt the $\mathrm{CL}$ wearer to use eyeglasses until resolution, discard or disinfect the $\mathrm{CL}$ if nondisposable.

\section{For Medical Personnel}

- Reschedule. Whenever possible, elective consultations and surgeries can be postponed. Routine annual checkups can be delayed for a few months. Recently, the AAO has released guidelines on resuming elective care, advising gradual return and emphasizing social distancing in waiting rooms, appropriate cleaning and disinfection, and protective examination gear and equipment.

- Screen. Check for fever, upper airway symptoms, or any newly noticeable systemic disease on arrival. Ask about recent travel or contact with infected cases. Any non-urgent cases with a risk of infection should be referred for medical attention and their visit postponed.

- Wear a mask. This is the recommendation for all visits. Infected patients or suspected carriers in need of urgent ophthalmic care should be examined in isolated rooms by physicians wearing full protective gear.

- Use a shield. While smaller breath shields are protective against large particles, recent studies have shown the importance of larger ones as more potent barrier protection [5]. However, studies, and personal experience, have found that these shields intervened with the manipulation of the slit-lamp and the applanation tonometer, and increased the risk of fomite transmission. Therefore, they should not substitute the use of masks and frequent hand hygiene. There are some readily available breath shields, but you can also create one yourself: https://crstodayeurope.com/articles/not-rated/slit-lampdroplet-shield/.

- Avoid talking during examination. Besides the usual gaze-directing remarks, avoid talking while examining the patient on the slit-lamp.

- Disinfect. Refer to the manual of all instruments to ensure proper disinfection while maintaining the integrity of the materials. Thoroughly disinfect the chin rest and forehead support, as well as the reusable Goldmann tips, between patients. When possible, use disposable instruments including tonometer prisms, single-use diagnostic lenses, sterile carriers for three-mirror contact lenses that act as a barrier with the tear film. Due to the uncertainty of the presence of viral load in tears, avoid puff tonometry since it could potentially generate aerosols. However, due to increasing evidence, the Royal College of Ophthalmologists has allowed non-contact tonometry in cases without active conjunctivitis provided that appropriate disinfection is done and that three puffs are performed after each patient.

- Test before surgery. This depends on individual risk factors and the availability of the test. Preoperative preparation with $5 \%$ povidone-iodine remains the standard of care. The presence of SARS-CoV-2 in 
aqueous or vitreous humor remains unknown. The risk of aerosolization with phacoemulsification is considered to be very low. The AAO recommends testing before the following surgeries: canalicular and nasolacrimal procedures; eyelid repair and inner approach ptosis repair; orbital tumors; anophthalmic socket surgery; and surgeries with manipulation of mucous membranes. In the absence of a negative PCR result for such patients, the AAO recommends wearing more protective equipment including an N95 mask and face shield due to the higher risk of aerosolization. External cautery should be preceded by povidone-iodine and accompanied by BSS irrigation. The risk with other surgeries is considered low.

Scientific research and optimal healthcare are of utmost importance during this period. Healthcare professionals and the public have a responsibility towards what the future unfolds.

\section{References}

1. Whittaker E, Bamford A, Kenny J, Kaforou M, Jones CE, Shah P, Ramnarayan P, Fraisse A, Miller O, Davies P, Kucera F, Brierley J, McDougall M, Carter M, Tremoulet A, Shimizu C, Herberg J, Burns JC, Lyall H, Levin M; PIMS-TS Study Group and EUCLIDS and PERFORM Consortia. Clinical Characteristics of 58 Children With a Pediatric Inflammatory Multisystem Syndrome Temporally Associated With SARS-CoV-2. JAMA. 2020 Jul 21;324(3):259-269. doi: 10.1001/jama.2020.10369. PMID: 32511692; PMCID: PMC7281356. https://doi.org/10.1001/jama.2020.10369

2. Zhou Y, Duan C, Zeng Y, Tong Y, Nie Y, Yang Y, Chen Z, Chen C. Ocular Findings and Proportion with Conjunctival SARS-COV-2 in COVID-19 Patients. Ophthalmology. 2020 Jul;127(7):982-983. doi: 10.1016/j.ophtha.2020.04.028. Epub 2020 Apr 21. PMID: 32359840; PMCID: PMC7194804. https://doi.org/10.1016/j.ophtha.2020.04.028

3. Seah IYJ, Anderson DE, Kang AEZ, Wang L, Rao P, Young BE, Lye DC, Agrawal R. Assessing Viral Shedding and Infectivity of Tears in Coronavirus Disease 2019 (COVID-19) Patients. Ophthalmology. 2020 Jul;127(7):977-979. doi: 10.1016/j.ophtha.2020.03.026. Epub 2020 Mar 24. PMID: 32291098; PMCID: PMC7151491. https://doi.org/10.1016/j.ophtha.2020.03.026

4. Mukamal R. Eye Care During the Coronavirus Pandemic (COVID-19) [Internet]. American Academy of Ophthalmology; 2020 May [cited 2020 Aug 2]. Available from: https://www.aao.org/eye-health/tips-prevention/coronavirus-covid19-eyeinfection-pinkeye

5. Poostchi A, Kuet ML, Pegg K, Wilde C, Richardson PS, Patel MK. Efficacy of slit lamp breath shields. Eye (Lond). 2020 Jul;34(7):1185-1186. doi: 10.1038/s41433-020-0940-y. Epub 2020 May 12. PMID: 32398843; PMCID: PMC7216572. https://doi.org/10.1038/s41433-020-0940-y 